\title{
miR-650 Promotes the Metastasis and Epithelial-Mesenchymal Transition of Hepatocellular Carcinoma by Directly Inhibiting LATS2 Expression
}

\author{
Li Li Han Xiao Ran Yin Shu Qun Zhang \\ Department of Oncology, The Second Affiliated Hospital of Xi'an Jiaotong University, Xi'an, China
}

\section{Key Words}

EMT • Hepatocellular carcinoma $・$ LATS2 $・$ miR-650 • YAP

\begin{abstract}
Background/Aims: Previous studies have confirmed that microRNAs are involved in the metastasis and epithelial-mesenchymal transition (EMT) of malignancies. In this study, we examined whether miR-650 promotes the migration, invasion, and EMT of hepatocellular carcinoma (HCC) cells by targeting the large tumor suppressor kinase 2 gene (LATS2). Methods: qRT-PCR was used to detect expression of miR-650 in HCC tissues and paired normal tissues. MTT and Transwell assay were used to observe the effect of miR-650 on proliferation, migration and invasion of HCC cells. Western blot assay and Immunohistochemistry were performed to demonstrate association between miR-650 expression level and epithelial-mesenchymal transition (EMT) related protein. Mechanistically, Reporter luciferase assay was performed to reveal whether large tumor suppressor kinase 2 (LATS2) was a direct target of miR-650 in HCC cells. Results: We observed that miR-650 levels were largely up-regulated in HCC tissues, and that the increased expression was closely associated with the adverse clinical features of HCC patients. Additionally, the expression of LATS2, which was identified as a direct target of miR-650, can counteract the effects of miR-650 in HCC. Furthermore, we demonstrated that high miR-650 expression levels and low LATS2 expression levels in tumors may indicate a poor prognosis for HCC patients. Conclusion: In conclusion, the miR-650/LATS2 pathway may serve as a novel prognostic biomarker and an attractive therapeutic target for HCC patients.
\end{abstract}




\section{Introduction}

Hepatocellular carcinoma (HCC) is one of the leading causes of cancer-related deaths worldwide [1]. The prognosis for HCC patients is currently extremely poor because of the highly invasive nature of the disease [2]. Additionally, the mechanisms underlying HCC metastasis have not been accurately characterized. The epithelial-mesenchymal transition (EMT), which involves the conversion of a cancer cell from its epithelial form to its motile mesenchymal form, is recognized as an initial and essential event in the metastatic progression of various carcinomas [3, 4]. MicroRNAs (miRNAs) are a class of small noncoding RNAs that serve as post-transcriptional regulators mainly by targeting and degrading specific mRNAs [5]. miRNAs function as crucial modulators that regulate many cancerrelated biological processes, including invasion, metastasis, and EMT [6]. Many miRNAs are involved in the metastasis and EMT of HCC. Recent research has focused on the effect of miR-650 on the progression of human cancers $[7,8]$. The miR-650 genomic sequence is associated with the Ig $\lambda$ variable region gene [9]. Interestingly, miR-650 may have different roles in diverse cancers. For example, in chronic lymphocytic leukemia, miR-650 is positively associated with a favorable prognosis [10], while miR-650 can promote the progression of gastric cancer by stimulating metastasis [11]. Additionally, Zheng et al [12]. reported that increased expression of miR-650 is associated with the progression of HCC. However, no causal relationship has been established between miR-650 and HCC metastasis and EMT. Therefore, further research into miR-650 functions related to HCC progression is necessary.

In this study, we analyzed HCC tissue samples and revealed that miR-650 functions as a prognostic indicator. The effects of miR-650 on the growth, migration, invasion, and EMT of HCC cells and the possible underlying mechanisms were also investigated.

\section{Materials and Methods}

\section{Patients and tissue samples}

The 130 tissue samples analyzed in this study were collected from HCC patients who underwent a surgical resection at The Second Affiliated Hospital of Xi'an Jiaotong University between March 2010 and March 2011. The clinical-pathological characteristics of all patients are listed in Table 1. None of the patients underwent prior chemotherapy or radiotherapy. Fresh HCC tissue samples and paired distant noncancerous tissue samples ( $\geq 2 \mathrm{~cm}$ from the margin) were immediately frozen in liquid nitrogen during the surgical resection and stored at $-80^{\circ} \mathrm{C}$ (for a western blot or qRTPCR assay) or in paraffin (for an immunohistochemistry [IHC] assay). This study was approved by the Ethics Committee of Clinical Research of Xi'an Jiaotong University.

\section{qRT-PCR assay}

Theprimersused in the qRTPCR assay were designed and synthesized by Takara (Dalian, China), and the sequences are provided in Table 2 . The qRTPCR assay was conducted in triplicate. The U6 RNA level was used as the internal control for miR-650, while glyceraldehyde 3-phosphate dehydrogenase

Table 1. Association between miR-650 expression and clinicalpathological features in HCC $(n=130)$

\begin{tabular}{|c|c|c|c|c|}
\hline \multirow{2}{*}{ Variable } & \multirow{2}{*}{ Total no. of patients $n=130$} & \multicolumn{2}{|c|}{ miR-650 } & \multirow[b]{2}{*}{$\mathrm{p}$} \\
\hline & & Low expression & High expression & \\
\hline Age (years) & & & & 0.348 \\
\hline$<50$ & $65(50.0 \%)$ & 24 & 41 & \\
\hline$\geq 50$ & $65(50.0 \%)$ & 18 & 47 & \\
\hline Gender & & & & 0.223 \\
\hline Female & $26(20.0 \%)$ & 11 & 15 & \\
\hline Male & $104(80.0 \%)$ & 31 & 73 & \\
\hline HBsAg & & & & 0.832 \\
\hline Positive & $107(82.3 \%)$ & 35 & 72 & \\
\hline Negative & $23(17.7 \%)$ & 7 & 16 & \\
\hline $\mathrm{AFP}(\mathrm{ng} / \mathrm{mL})$ & & & & 0.272 \\
\hline$<400$ & $53(40.8 \%)$ & 20 & 33 & \\
\hline$\geq 400$ & $77(59.2 \%)$ & 22 & 55 & \\
\hline Cirrhosis & & & & 0.554 \\
\hline Yes & $76(58.5 \%)$ & 23 & 53 & \\
\hline No & $54(41.5 \%)$ & 19 & 35 & \\
\hline Tumor size $(\mathrm{cm})$ & & & & $0.003^{* *}$ \\
\hline$<5$ & $75(57.7 \%)$ & 32 & 43 & \\
\hline$\geq 5$ & $55(42.3 \%)$ & 10 & 45 & \\
\hline Tumor multiplicity & & & & 0.647 \\
\hline Single & $111(85.4 \%)$ & 35 & 76 & \\
\hline Multiple & $19(14.6 \%)$ & 7 & 12 & \\
\hline Differentiation & & & & 0.885 \\
\hline Well-moderate & $60(46.2 \%)$ & 19 & 41 & \\
\hline Poor-undifferentiation & $70(53.8 \%)$ & 23 & 47 & \\
\hline Microscopic vascular invasion & & & & $<0.001^{* *}$ \\
\hline Yes & $49(37.7 \%)$ & 26 & 23 & \\
\hline No & $91(62.3 \%)$ & 16 & 65 & \\
\hline Stage & & & & $0.043^{*}$ \\
\hline I-II & $83(63.8 \%)$ & 32 & 51 & \\
\hline III-IV & $47(36.2 \%)$ & 10 & 37 & \\
\hline
\end{tabular}




\section{Cellular Physiology Cell Physiol Biochem 2018;51:1179-1192 and Biochemistry Publisned ontune:247November $2018 \begin{aligned} & \text { (c) } 2018 \text { The Author(s). Published by S. Karger AG, Basel } \\ & \text { www.karger.com/cpb }\end{aligned}$}

Han et al.: miR-650 Promotes the EMT of HCC by Directly Inhibiting LATS2

$(G A P D H)$ was used as the internal control for large tumor suppressor kinase 2 (LATS2) and the other genes. The gene expression levels relative to the $G A P D H$ expression level were assessed using the $2^{-\Delta \Delta \mathrm{ct}}$ method.
Table 2. Sequences of the qRT-PCR primers

\begin{tabular}{lcc}
\hline Variable & \multicolumn{1}{c}{ Forward } & Reverse \\
\hline miR-650 & 5'-AGAGGAGGCAGCGCTCT-3' & 5'-CAGTGCGTGTCGTGGAGT-3' \\
LATS2 & 5'-ACCCCAAAGTTCGGACCTTAT-3' & 5'-CATTTGCCGGTTCACTTCTGC-3' \\
Cyr61 & 5'-CCCTGAACTTGTGGATGTCATTG-3' & 5'-GTCATGATGATCCAGTCCTGCAAA-3' \\
AREG & 5'-TGCTGGATTGGACCTCAATG-3' $^{\prime}$ & 5'-TCCCGAGGACGGTTCACTAC-3' $^{\prime}$ \\
CTGF & 5'-GAAAAGAUUCCCACCCAU-3' $^{\prime}$ & 5'-AUUGGGUGGGAAUCUUUUC-3' $^{\prime}$ \\
CXCL5 & 5'-GTTCCATCTCGCCATTCATGC-3' & 5'-GCGGCTATGACTGAGGAAGG-3' \\
GAPDH & 5'-AATGGACAACTGGTCGTGGAC-3' & 5'-CCCTCCAGGGGATCT GTTTG-3' \\
\hline
\end{tabular}

IHC assay

An IHC assay was performed as previously described [13]. The antibodies used in this study included the anti-LATS2 antibody ( bs-4081R, Beijing Bioss Biotechnology, Beijing, China), anti-E-cadherin antibody (cat. bs-1016R, Beijing Bioss Biotechnology), and anti-vimentin antibody (cat. bs-8533R, Beijing Bioss Biotechnology).

\section{Cell culture and transfection}

The HCC and LO2 cell lines were purchased from the American Type Culture Collection (Manassas, VA) and were cultured in Dulbecco's modified Eagle's medium supplemented with $10 \%$ fetal bovine serum (FBS), $100 \mu \mathrm{g} / \mathrm{ml}$ streptomycin, and $100 \mathrm{units} / \mathrm{ml}$ penicillin (Sigma-Aldrich, St. Louis, MO) in an incubator set at $37^{\circ} \mathrm{C}$ with $5 \% \mathrm{CO}_{2}$. miR-650 mimic was acquired from Genecopoeia (Guangzhou, China), while the LATS2 vector was obtained from Addgene (Cambridge, MA). Additionally, an anti-miR-650 inhibitor and LATS2 small interfering RNA (siRNA) were purchased from Origene (Beijing, China). Cells were transfected with these vectors and siRNA using Lipofectamine 2000 (Thermo Fisher Scientific, Waltham, MA) according to the manufacturer's protocol.

\section{Western blot assay}

The cells of each line were lysed in RIPA Buffer (cat. 9800, Cell Signaling Technology, Danvers, MA) to extract proteins, after which the protein concentrations were determined in bicinchoninic acid assays (cat. 7780, Cell Signaling Technology). For each sample, the total protein content was quantified with the Bradford method. Total proteins $(25 \mu \mathrm{g})$ were resolved by $12 \%$ sodium dodecyl sulfate-polyacrylamide gel electrophoresis and subsequently transferred to polyvinylidene fluoride membranes. The membranes were blocked in 5\% non-fat milk for $1 \mathrm{~h}$ and then probed with the following primary antibodies: antiLATS2 antibody (cat. bs-4081R; Beijing Bioss Biotechnology, Beijing, China), anti-yes-associated protein (YAP) antibody (cat. 14074, Cell Signaling Technology), anti-phospho-YAP (Ser127) antibody (cat. 13008, Cell Signaling Technology), anti-E-cadherin antibody (cat. 3195, Cell Signaling Technology), anti-vimentin antibody (cat. 5741, Cell Signaling Technology), and anti-GAPDH (cat. ab6922; Abcam, MA, USA). The membranes were incubated at $4^{\circ} \mathrm{C}$ overnight, washed, and then incubated with anti-rabbit secondary antibodies for LATS2, YAP, phosphorylated YAP (Ser127), E-cadherin, vimentin, and an anti-mouse secondary antibody for GAPDH at $37^{\circ} \mathrm{C}$ for $2 \mathrm{~h}$. Finally, the membranes were analyzed with a bio-imaging system (DNR Bio-Imaging Systems, Jerusalem, Israel).

\section{Cell migration and invasion assays}

The migration and invasive ability of HCC cells were measured in 24-well Transwell plates (Corning Costar, Tewksbury, MA). For the cell migration assays, $2 \times 10^{4}$ HCC cells in $200 \mu \mathrm{l} \mathrm{FBS-free} \mathrm{medium} \mathrm{were}$ uniformly implanted in the upper chamber, while $600 \mu$ normal culture medium (with 10\% FBS) was added to the bottom chamber. After a 48-h incubation, the cells remaining on the membrane in the upper chamber were carefully removed with a cotton swab, while the cells that migrated through the membrane to the surface of the bottom chamber were gently cleaned and fixed with methanol. The migratory cells were stained with crystal violet solution and air-dried at $37^{\circ} \mathrm{C}$. The migratory cells in 10 random microscopic fields were counted. For the invasion assay, we performed the same procedures as described above except we also coated the membrane in the upper chamber with $200 \mathrm{mg} / \mathrm{ml}$ Matrigel (BD Biosciences, San Jose, CA). 


\section{Cellular Physiology Cell Physiol Biochem 2018;51:1179-1192 and Biochemistry \begin{tabular}{l|l} 
DOI: 10.1159/000495495 2018 The Author(s). Published by S. Karger AG, Basel \\
Publor 2018
\end{tabular}

\section{MTT assays}

MHCC97-H or HepG2 cells (500 cells/well in $100 \mu \mathrm{l}$ medium) were added to a 96-well plate and incubated at $37^{\circ} \mathrm{C}$ for $24,48,72,96,120,144$, and $168 \mathrm{~h}$. The medium was replaced with fresh medium every $48 \mathrm{~h}$ for samples incubated for $72,96,120,144$, and $168 \mathrm{~h}$. At each analyzed time point, $10 \mu \mathrm{l}$ MTT solution ( $5 \mathrm{mg} / \mathrm{ml}$ in PBS) was added to each well. After a 4 -h incubation at $37^{\circ} \mathrm{C}$, the medium was removed, and 200 $\mu \mathrm{l}$ dimethyl sulfoxide was added. Cell viability was assessed using a plate reader (492 nm wavelength) (BioRad Laboratories, Hercules, CA). Analyses were conducted in triplicate.

\section{Luciferase reporter assay}

The indicated cells were added to a 24-well plate and transfected with miR-650, the control, or 3 '-untranslated region (UTR)-luciferase plasmids. Cells were collected at $48 \mathrm{~h}$ after the transfection, and luciferase activity was measured using a Dual-Luciferase Assay System (Promega, Madison, WI).

\section{Statistical analysis}

Statistical analyses were performed using SPSS 20.0 (SPSS Inc., Chicago, IL). All experiments were repeated three times. The data are presented as the mean \pm standard deviation. The chi-square test and Fisher's exact test (two-sided) were conducted to assess the association between clinical-pathological characteristics and gene expression. Spearman correlation analysis was used to examine the correlation between the expression levels of two genes. The Kaplan-Meier method (log-rank test) was completed to plot the survival curves of different sub-groups. A p-value less than 0.05 in a two-sided test was applied as the threshold for significance.

\section{Results}

Overexpression of miR-650 is associated with HCC progression

qRT-PCR analysis revealed that miR-650 expression levels were considerably higher in HCC tissue samples than in the paired normal tissues (5.38 \pm 0.33 vs. $2.98 \pm 0.12, p<0.01$, Fig. 1A). A subsequent analysis suggested that miR-650 expression was significantly higher in HCC tissues from patients with metastasis than in patients without metastasis $(6.12 \pm$ 0.21 vs. $4.18 \pm 0.17, p<0.01$, Fig. $1 B$ ). We also evaluated the clinical significance of miR-650 in HCC patients. The data indicated that high miR-650 expression levels were associated with microscopic vascular invasion $(p<0.001)$, a relatively large tumor $(p=0.003)$, and an advanced TNM stage in HCC patients ( $\mathrm{p}=0.043$ ) (Table 1 ).

A

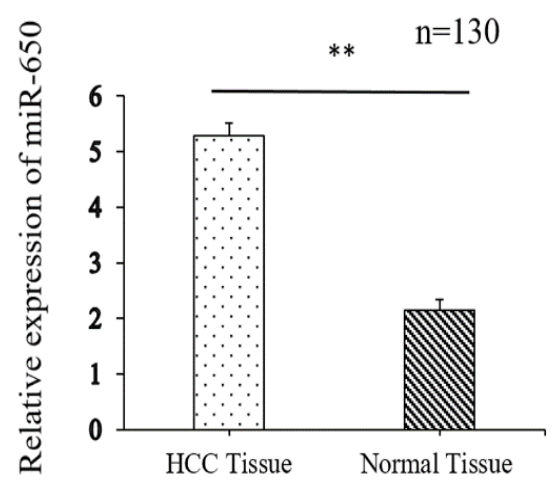

B

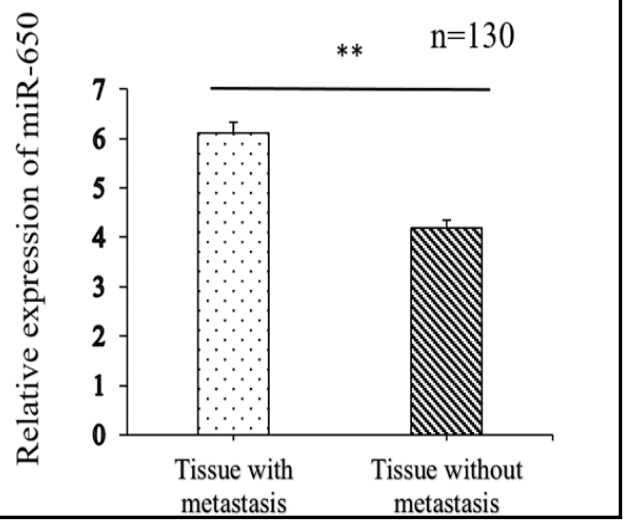

Fig. 1. Expression of miR-650 in HCC tissues. A. Relative miR-650 expression levels were compared between HCC tissues and paired distant non-cancerous tissues $\left(n=130,{ }^{* *} p<0.01\right)$. B. Comparison of relative miR650 expression levels in tumor samples from patients with and without metastasis $(\mathrm{n}=130, * * \mathrm{p}<0.01)$. Target gene expression levels were quantified relative to GAPDH expression levels. 


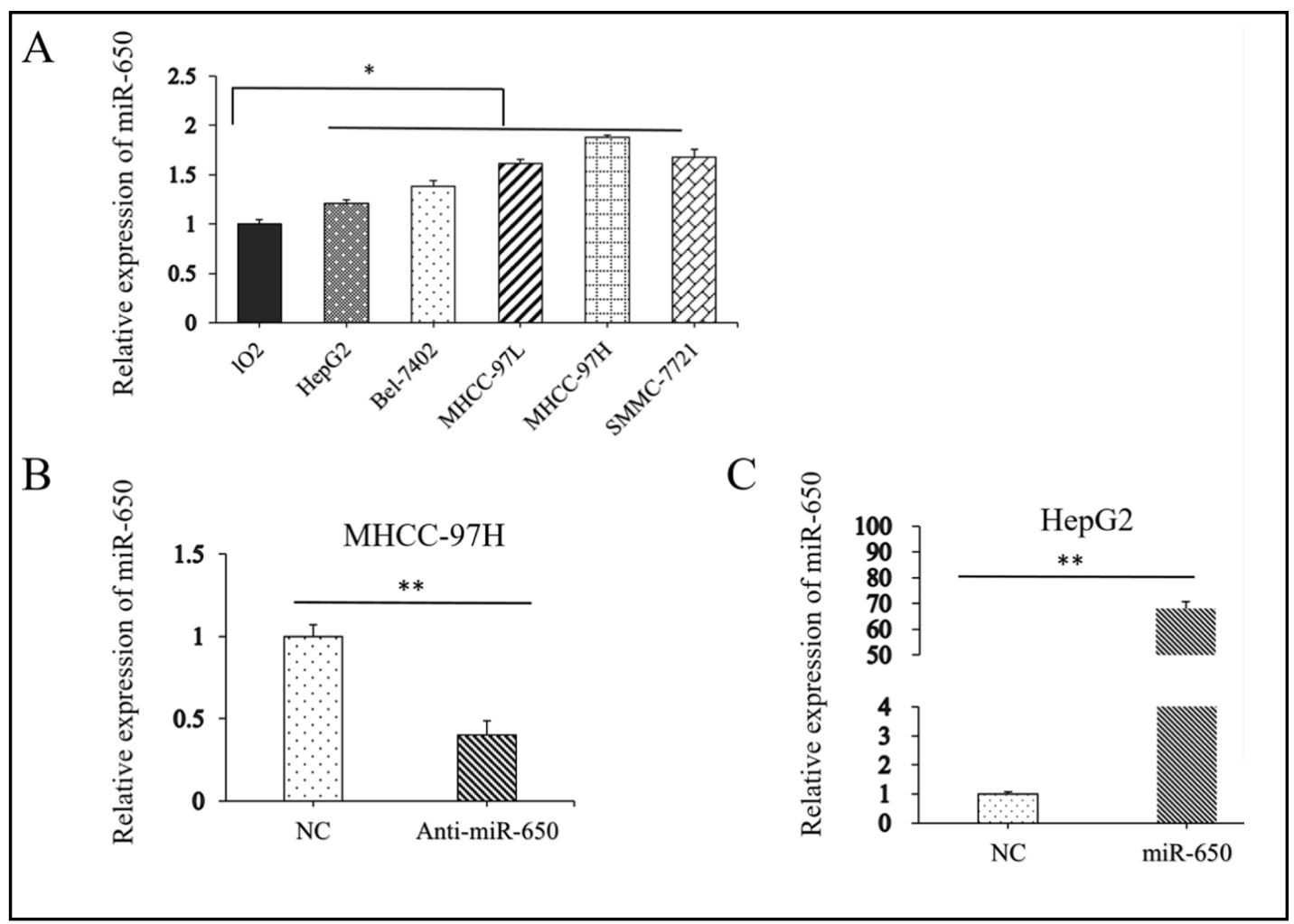

Fig. 2. Expression of miR-650 in HCC cells. A. miR-650 expression levels in various human HCC cell lines and human hepatocyte LO2 cells $\left({ }^{*} \mathrm{p}<0.05\right)$. B. miR-650 expression level significantly decreased after MHCC$97 \mathrm{H}$ cells were transfected with the miR-650 inhibitor $\left({ }^{* *} \mathrm{p}<0.01\right)$. C. miR-650 expression level significantly increased after HepG2 cells were transfected with the miR-650 mimic $(* * p<0.01)$.

miR-650 promotes HCC cell migration and invasion

To evaluate the function of miR-650 in HCC cells, we compared the miR-650 expression levels in HCC cell lines and the human liver cell line L02. Consistent with the data for the HCC samples, the qRT-PCR analysis confirmed that miR-650 expression levels were markedly higher in HCC cells than in LO2 cells ( $p<0.05$, Fig. 2A). Among the HCC cell lines, the highest and lowest miR-650 expression levels were observed in MHCC-97H and HepG2 cells, respectively. We then transfected MHCC97-H and HepG2 cells with the miR-650 inhibitor and the miR-650 mimic, respectively. The miR-650 inhibitor decreased the miR-650 expression level in MHCC97-H cells ( $\mathrm{p}<0.01$, Fig. 2B), while the miR-650 mimic considerably increased the miR-650 expression level in HepG2 cells ( $<<0.05$, Fig. 2C). Analyses of cell migration and invasion suggested that decreased miR-650 levels markedly inhibited the migration and invasion capacity of MHCC-97H cells ( $p<0.01$, Fig. 3A). In contrast, the increased miR-650 expression in HepG 2 cells significantly enhanced the migration and invasion ability of cells ( $p<0.01$, Fig. 3B). Our results demonstrated that miR-650 promotes HCC cell migration and invasion.

\section{miR-650 promotes HCC EMT}

We further investigated whether miR-650 helps mediate HCC EMT. The western blot data revealed that decreased miR-650 levels resulted in increased E-cadherin expression (epithelial marker) and decreased vimentin and Snail expression (mesenchymal markers) in MHCC-97H cells. The opposite trends were observed when miR-650 levels increased $(\mathrm{p}<$ 0.01 , Fig. 4A). To evaluate whether miR-650 promotes HCC EMT, we conducted an IHC assay to compare the E-cadherin and vimentin levels in HCC tissues ( $p<0.01$, Fig. 4B). Spearman 


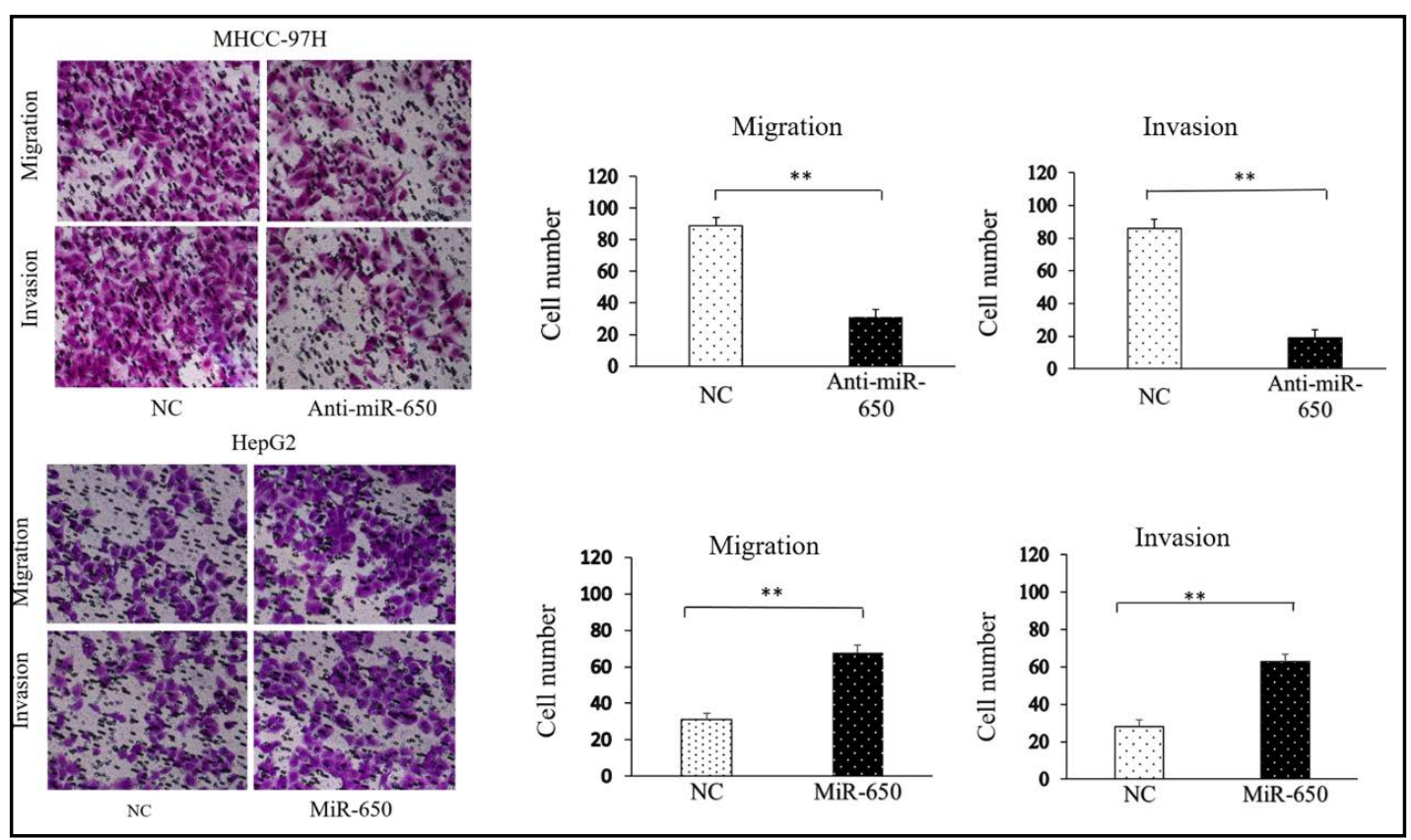

Fig. 3. Analysis of cell migration and invasion using 24-well Transwell plates indicated miR-650 expression affected HCC cell invasion and migration. The invaded cells were quantified by counting the cells in 10 random fields (magnification 200x). Data are presented as the mean \pm standard deviation of three independent experiments.

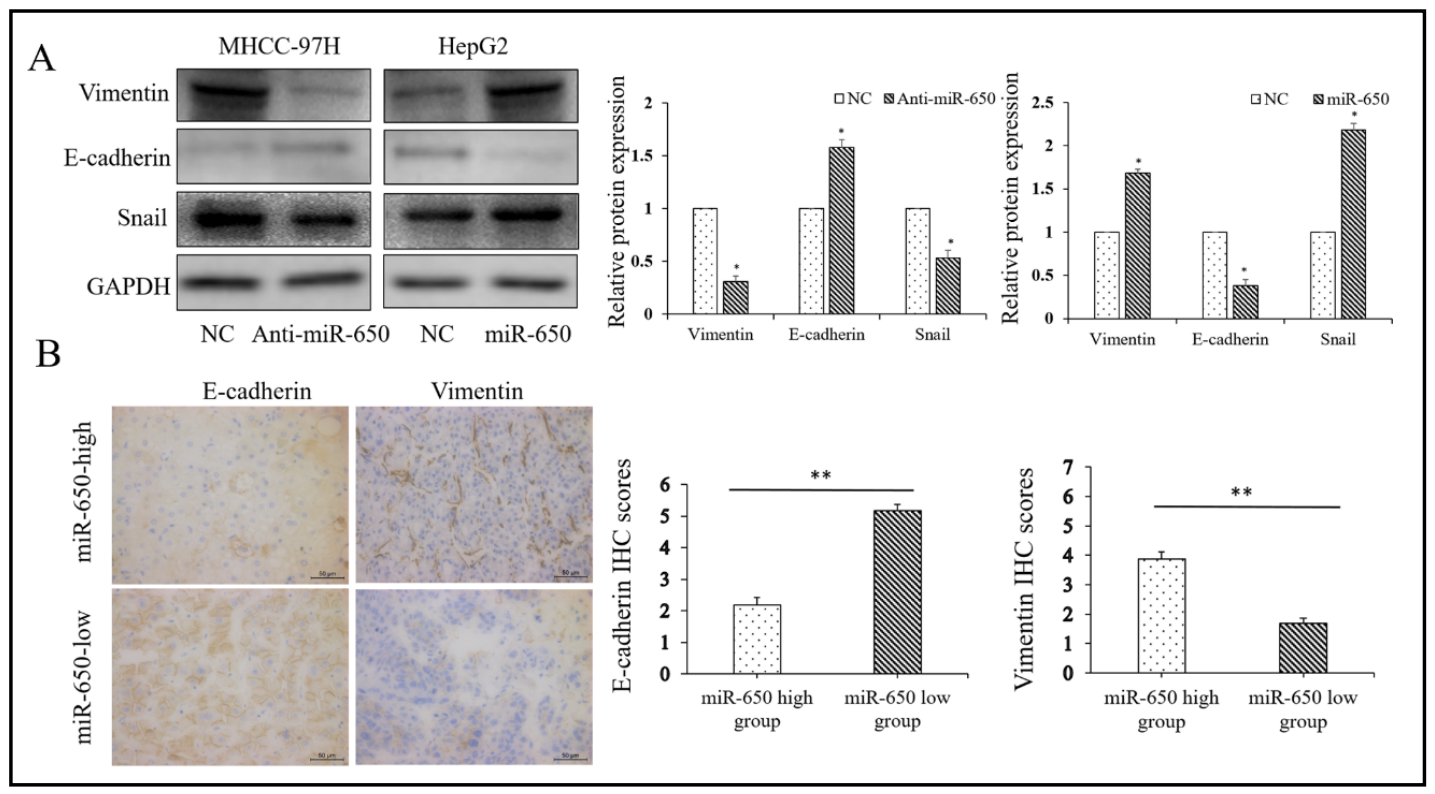

Fig. 4. miR-650 promotes HCC EMT. A. Western blot analysis of E-cadherin, vimentin, and Snail in MHCC97H cells in which miR-650 expression was down-regulated and HepG2 cells in which miR-650 expression was up-regulated $(* \mathrm{p}<0.05)$. B. IHC analysis of E-cadherin and vimentin levels in tissues with high and low miR-650 expression levels $(n=130, * p<0.01)(400 \times)$. 
correlation analysis confirmed that the miR-650 expression level was negatively associated with the E-cadherin expression level $(\mathrm{p}<0.001, \mathrm{r}=-0.468)$, but positively associated with the vimentin expression level $(\mathrm{p}<0.001, \mathrm{r}=0.275$; Table 3$)$. These results implied that miR650 promotes HCC EMT.

\section{LATS2 may be directly targeted by miR-650 in HCC cells}

To explore the potential mechanism underlying the miR-650 function in HCC cells, we searched the TargetScan public database for genes targeted by miR-650. We detected a sequence complementary to miR-650 in the 3'-UTR of LATS2 mRNA (Fig. 5A). Because LATS2 is a critical regulator of cancer metastasis via its effects on the Hippo signaling pathway, which is important for regulating EMT [14], we examined whether miR-650 promotes HCC EMT through LATS2. Specifically, we performed an IHC assay to assess the LATS2 abundance in HCC tissues and paired distant non-cancerous tissues. We observed that LATS2 levels were markedly lower in HCC tissues than in non-cancerous tissues $(2.14 \pm 0.12$ vs. $4.83 \pm 0.24, \mathrm{p}<$ 0.01 , Fig. 5B). Moreover, LATS2 levels were considerably lower in samples from patients with metastasis than in samples from

Table 3. Association between miR-650 and EMT-related protein expression in HCC tissue samples. ${ }^{*} \mathrm{p}<0.05 ;{ }^{* *} \mathrm{p}<0.01 ; \mathrm{n}=130$

\begin{tabular}{lcccc}
\hline & \multicolumn{2}{c}{ miR-650 } & & \\
Variable & $\begin{array}{l}\text { High } \\
\text { expression }\end{array}$ & $\begin{array}{c}\text { Low } \\
\text { expression }\end{array}$ & r & $p$ \\
\hline LATS2 & & & & \\
Tumor & 30 & 28 & -0.306 & $<0.001^{* *}$ \\
High expression & 30 & 14 & & \\
Low expression & 58 & & -0.122 & 0.167 \\
Normal & & 34 & & \\
High expression & 29 & 28 & & \\
Low expression & 39 & & & \\
\hline
\end{tabular}

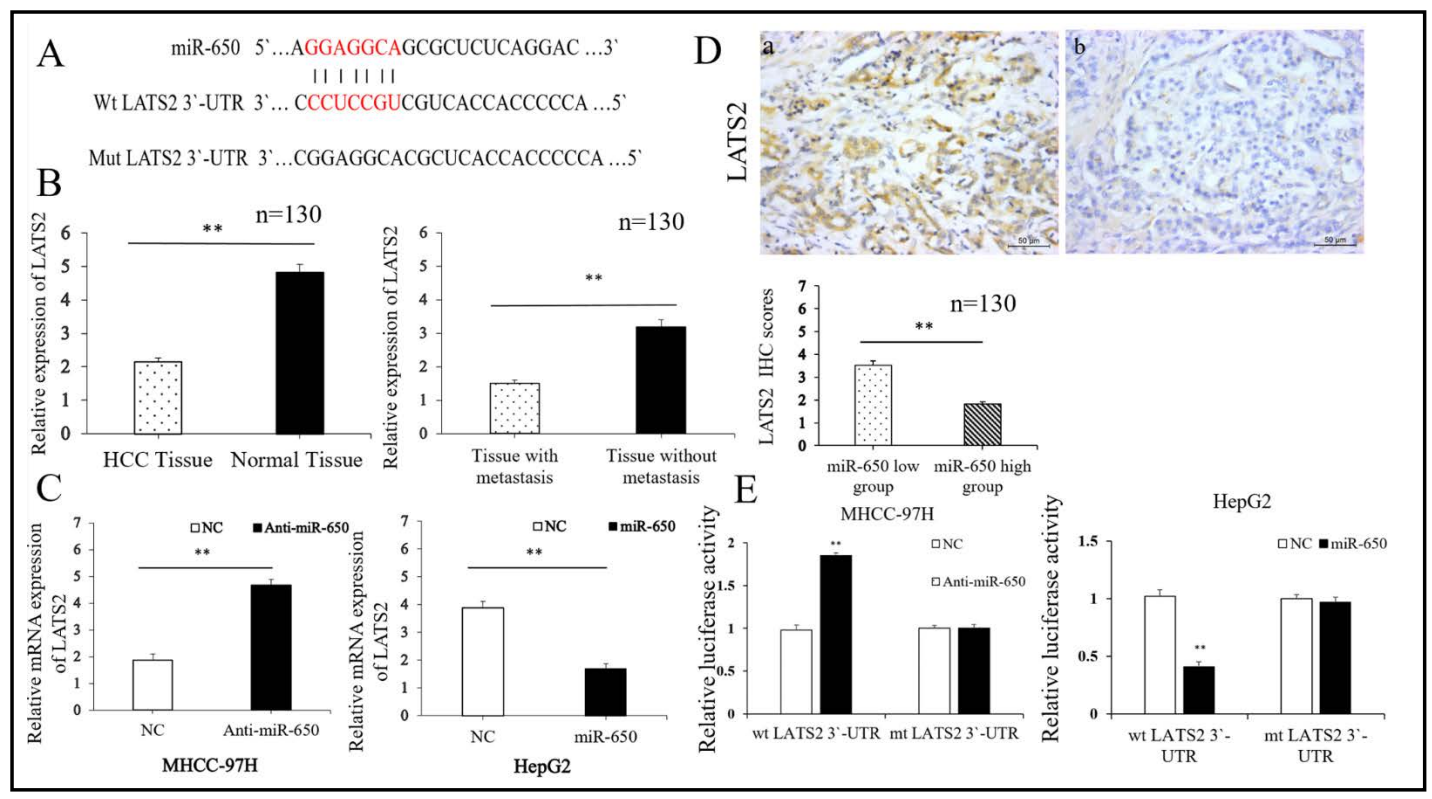

Fig. 5. LATS2 is a direct downstream target of miR-650 in HCC. A. miR-650 and its putative binding sequence in the $3^{\prime}$-UTR of LATS2. B. Comparison of relative LATS2 expression levels in HCC tissues and distant non-cancerous tissues $\left(\mathrm{n}=130,{ }^{* *} \mathrm{p}<0.01\right)$. Comparison of relative LATS2 expression levels in tumor samples from patients with and without metastasis $\left(n=130,{ }^{* *} p<0.01\right)$. C. LATS2 expression level significantly increased after MHCC-97H cells were transfected with the miR-650 inhibitor $(* * p<0.01)$. The LATS2 expression level significantly decreased after HepG2 cells were transfected with the miR-650 mimic $\left({ }^{* *} \mathrm{p}<0.01\right)$. D. IHC analysis of LATS2 in tissues with low (a) and high (b) miR-650 levels $\left(\mathrm{n}=130,{ }^{*} \mathrm{p}<0.01\right)$. E. Increased or decreased miR-650 levels significantly affected luciferase activity in HCC cells carrying wildtype LATS2, but not in HCC cells carrying LATS2 with a mutated $3^{\prime}$-UTR (**p<0.01). 
patients without metastasis $(1.51 \pm 0.09$ vs. $3.19 \pm 0.21, \mathrm{p}<0.01$, Fig. $5 B)$. Furthermore, we completed a qRT-PCR assay to examine the LATS2 expression level in miR-650 knockdown and overexpression HCC cells. Decreased miR-650 levels in MHCC-97H cells up-regulated LATS2 expression. However, increased miR-650 levels in HepG2 cells markedly down-regulated LATS2 expression ( $p<0.01$, Fig. 5C). Additionally, the IHC assay results (Fig. 5D) also indicated that LATS2 levels were markedly lower in HCC tissues in which miR-650 was highly expressed than in HCC tissues in which miR-650 was expressed at relatively low levels $(p<0.01)$. Statistical analysis revealed an inverse correlation between a high miR-650 expression level and a low LATS2 expression level in HCC tissue samples $(\mathrm{r}=-0.306)$. In contrast, a significant inverse association was not observed between LATS2 and miR-650 expression levels in noncancerous tissue samples ( $\mathrm{p}>0.05$; Table 4 ). To confirm that miR-650 binds to LATS2 to exert its effects in HCC cells, we completed luciferase reporter assays. Decreased miR-650 levels markedly promoted the luciferase activity associated with LATS2 with a wild-type 3'-UTR in MHCC-97H cells (Fig. 5E). Meanwhile, increased miR-650 levels in HepG2 cells inhibited the luciferase activity associated with LATS2 with a wild-type 3'-UTR. Additionally, altering miR-650 expression did not significantly affect the luciferase activity associated with LATS2 with a mutated $3^{\prime}$-UTR. These findings suggested that LATS2 may be directly targeted by miR650 in HCC cells.

Table 4. Association between miR-650 and LATS2 expression in HCC tissue samples. ${ }^{*} \mathrm{p}<0.05 ;{ }^{* *} \mathrm{p}<0.01 ; \mathrm{n}=130$

\begin{tabular}{|c|c|c|c|c|}
\hline \multirow[b]{2}{*}{ Variable } & \multicolumn{2}{|c|}{ miR-650 } & \multirow[b]{2}{*}{$\mathrm{r}$} & \multirow[b]{2}{*}{$\mathrm{p}$} \\
\hline & $\begin{array}{c}\text { High } \\
\text { expression }\end{array}$ & $\begin{array}{c}\text { Low } \\
\text { expression }\end{array}$ & & \\
\hline E-cadherin & & & -0.468 & $\mathrm{p}<0.001^{* *}$ \\
\hline High expression & 20 & 31 & & \\
\hline Low expression & 68 & 11 & & \\
\hline Vimentin & & & & \\
\hline High expression & 61 & 16 & 0.297 & $0.001^{*}$ \\
\hline Low expression & 27 & 26 & & \\
\hline
\end{tabular}

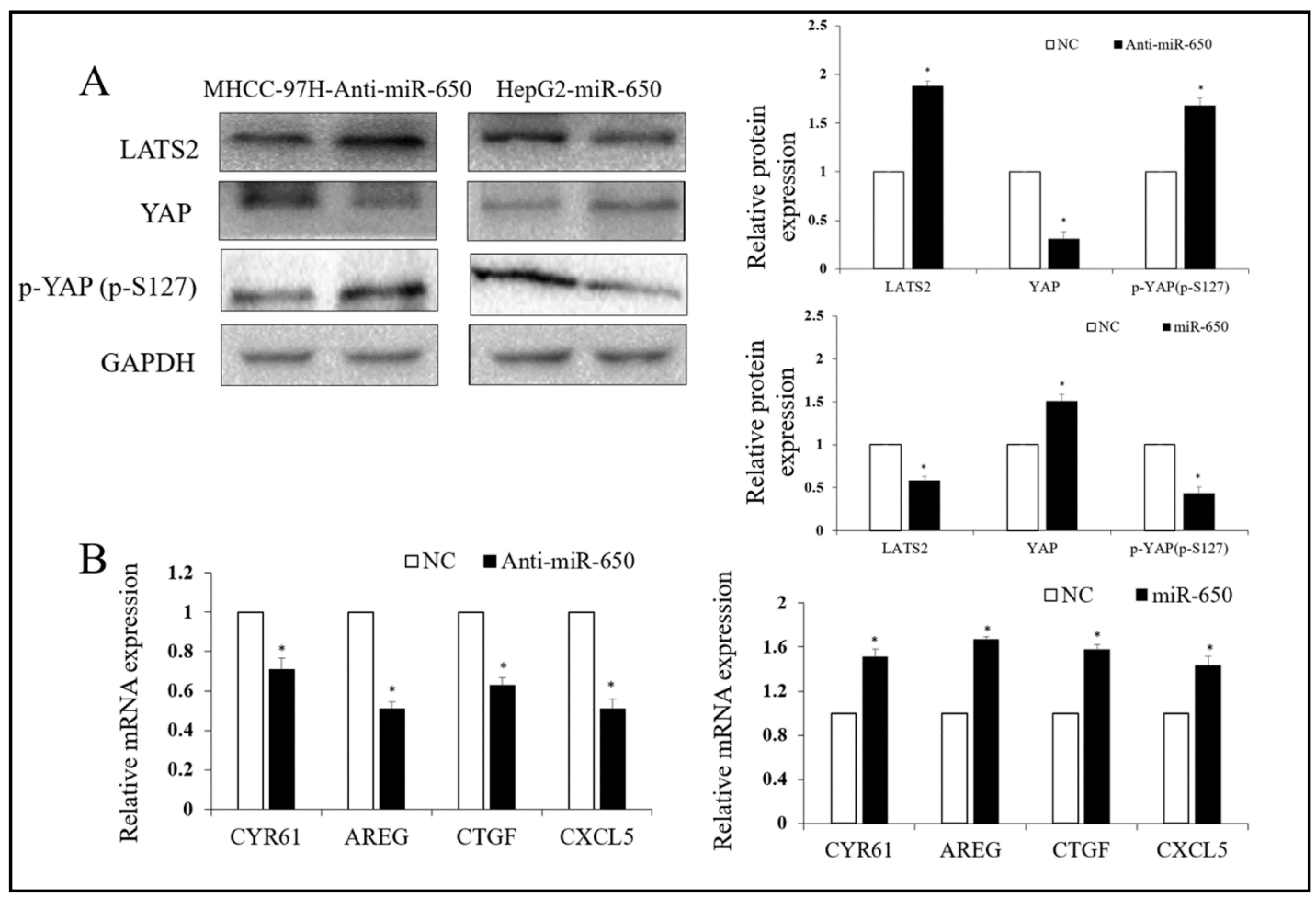

Fig. 6. Changes in miR-650 expression regulated the LATS2/YAP signaling pathway. A. Down-regulation or overexpression of miR-650 regulated the abundance of LATS2, YAP, and phosphorylated YAP (p-YAP [pS127]) ( $\left.{ }^{*} p<0.05\right)$. B. qRT-PCR analysis revealed that down-regulation or overexpression of miR-650 affected the expression of downstream YAP target genes, including CYR61, AREG, CTGF, and CXCL5 ( $\left.{ }^{\mathrm{p}}<0.05\right)$. 
MiR-650 regulates YAP and its downstream target genes

A previous study revealed that the LATS2 kinase phosphorylates the Hippo downstream effector YAP to regulate HCC EMT [15]. Therefore, we explored the effect of miR-650 on YAP by measuring the LATS2, YAP, and phosphorylated YAP (Ser127) levels. Decreased miR-650 levels significantly increased LATS2 levels (Fig. 6A). In contrast, miR-650 overexpression significantly decreased LATS2 levels ( $p<0.05$, Fig. 6A). Additionally, the phosphorylated YAP (Ser127) levels were measured using phospho-specific antibodies that recognize the LATS2 phosphorylation site on YAP (Ser127) [16]. Decreased miR-650 levels considerably increased the phosphorylated YAP (Ser127) levels, while increased miR-650 levels had the opposite effect ( $<<0.05$, Fig. 6A). Furthermore, qRT-PCR analysis indicated that some downstream YAP target genes (CYR61, AREG, CXCL5, and CTGF) were also regulated by miR650 (Fig. 6B). Thus, we demonstrated that miR-650 serves as an upstream LATS2/YAP signal that helps regulate YAP and its downstream target genes.

\section{LATS2 counteracts the miR-650 function in HCC cells}

To evaluate the effects of LATS 2 on the ability of miR-650 to promote metastasis, growth, and HCC EMT, MHCC-97H cells in which miR-650 levels were down-regulated and HepG2 cells in which miR-650 levels were up-regulated were treated with LATS2 siRNA and the LATS2 plasmid, respectively. Decreased LATS2 levels lessened the inhibition of the EMT due to the miR-650 inhibitor in MHCC-97H cells ( $p<0.05$ ) (Fig. 7A). Meanwhile, increased LATS2 levels reversed the promotion of EMT induced by miR-650 overexpression in HepG2 cells ( $p$ $<0.05$, Fig. 7A).

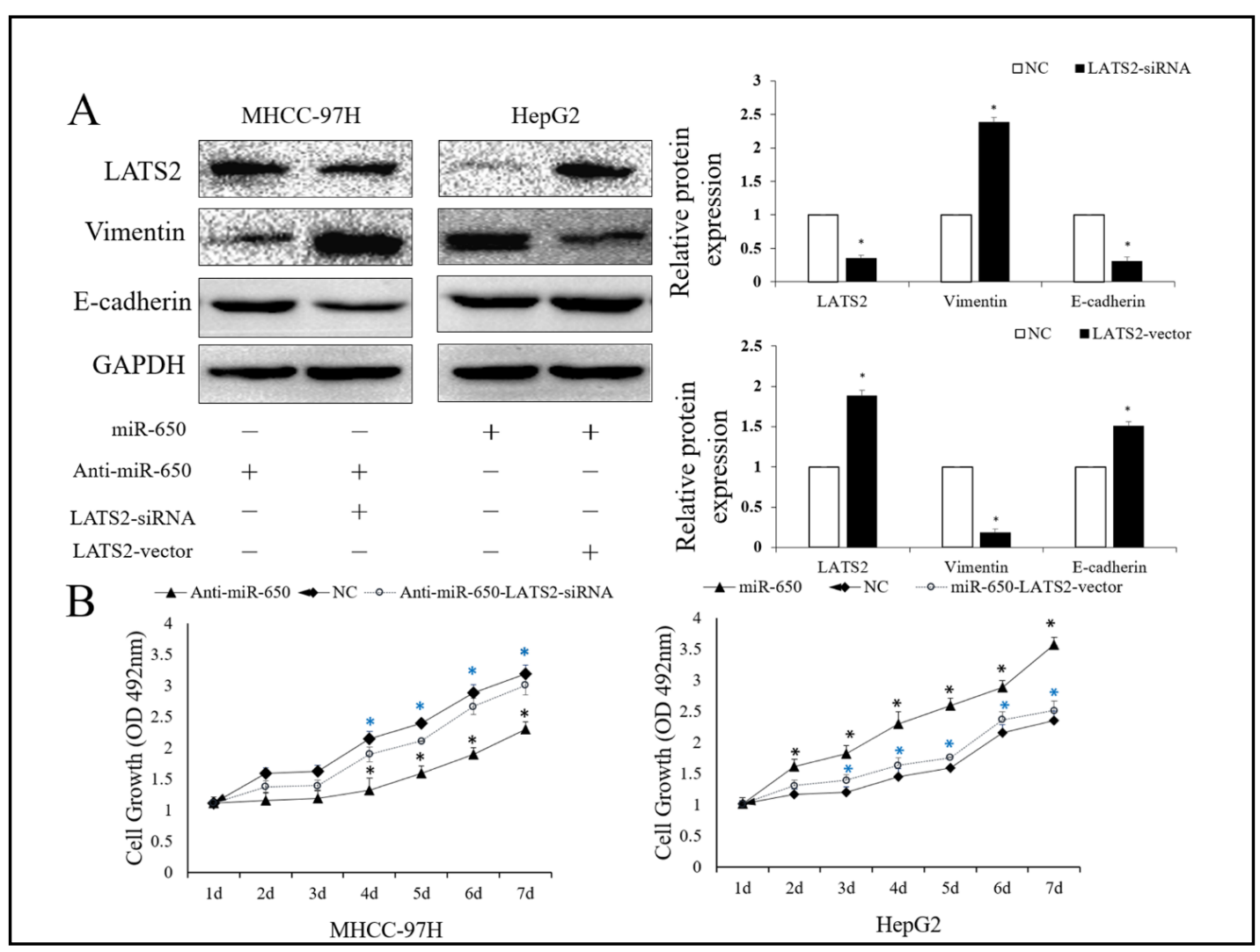

Fig. 7. LATS 2 mediates the functions of miR-650 in HCC cells $\left({ }^{*} \mathrm{p}<0.05\right)$. A. Western blot analysis indicated LATS2 mediates the effects of miR-650 on HCC EMT $\left({ }^{*} p<0.05\right)$. B. MTT assay confirmed that MHCC-97H cell proliferation decreased after miR-650 expression was down-regulated, while HepG2 cell proliferation increased after miR-650 expression was up-regulated $\left({ }^{*} \mathrm{p}<0.05\right)$. LATS2 mediates the effects of miR-650 on HCC cell proliferation $\left({ }^{*} \mathrm{p}<0.05\right)$. 


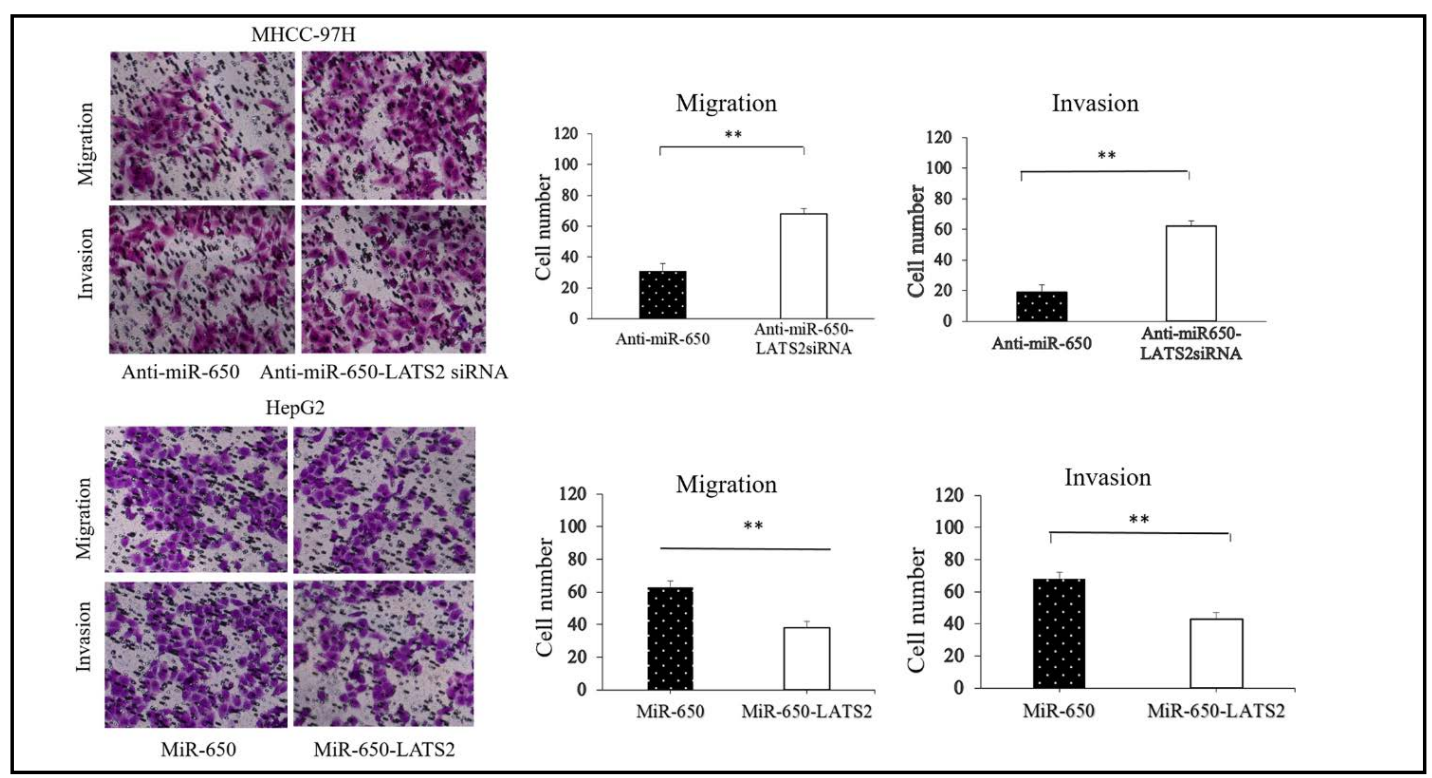

Fig. 8. Analysis of cell migration and invasion using 24-well Transwell plates revealed LATS2 mediates the effects of miR-650 on HCC cell migration and invasion $\left({ }^{*} \mathrm{p}<0.01\right)$. The invaded cells were quantified by counting the cells in 10 random fields (magnification 200×). Data are presented as the mean \pm standard deviation of three independent experiments.

Our MTT assay revealed that decreased miR-650 levels in MHCC-97H cells resulted in a diminished ability of cells to proliferate. In contrast, increased miR-650 levels in HepG2 cells significantly enhanced cell proliferation. The MTT assay also indicated that altering LATS2 expression could counteract the cell proliferation induced by miR-650 ( $p<0.01$, Fig. 7B).

Analyses of cell migration and invasion indicated that decreased LATS2 levels diminished the inhibited cell migration and invasion induced by decreased miR-650 levels in MHCC-97H cells, while increased LATS2 levels rescued the cell migration and invasion ability of HepG2 cells promoted by increased miR-650 levels ( $p<0.01$, Fig. 8). These results implied that LATS2 is an important determinant for the properties of miR-650 in HCC metastasis and EMT.

Prognostic significance of miR-650 and LATS2 expression for HCC patients

We evaluated the prognostic significance of miR-650 and LATS2 expression in HCC patients. An analysis using the Kaplan-Meier method revealed that high miR-650 expression levels were closely correlated with shorter overall survival (OS) and disease-free survival (DFS) ( $<<0.01$, Fig. 9A and B). Additionally, LATS2 expression was significantly associated with improved OS and DFS ( $\mathrm{p}<0.01$, Fig. 9C and D). Further analyses confirmed that HCC patients with high miR-650 expression levels and low LATS2 expression levels had the worst OS and DFS. In contrast, simultaneously low miR-650 expression levels and high LATS2 expression levels resulted in the best OS and DFS for HCC patients ( $\mathrm{p}<0.01$, Fig. 9E and F). Thus, the Kaplan-Meier analysis revealed a combination of miR-650 and LATS2 expression levels may be useful for determining the prognosis for HCC patients. 
A

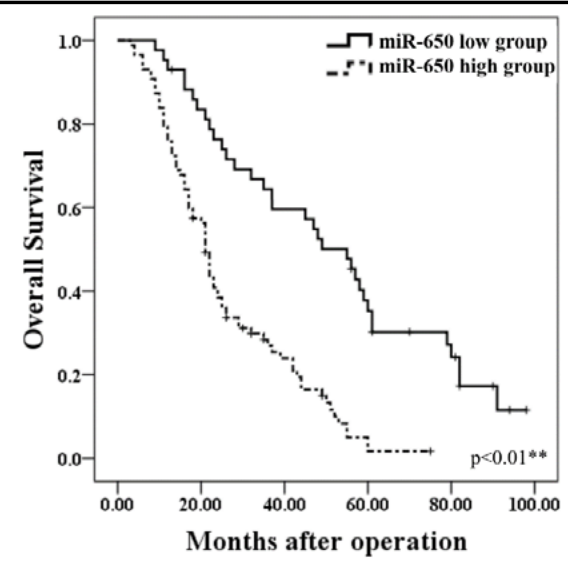

$\mathrm{C}$

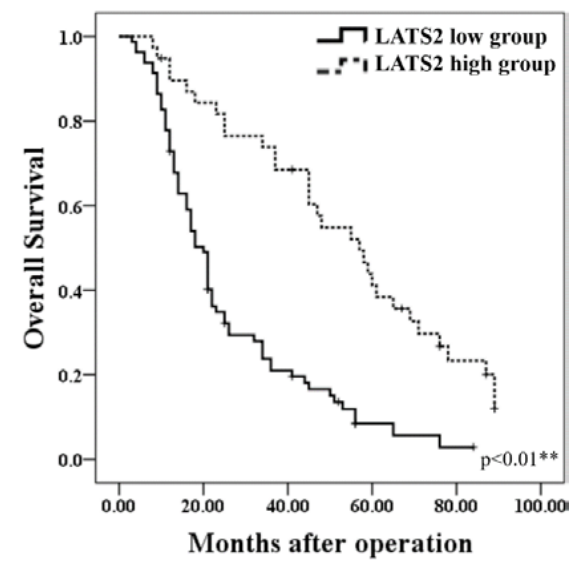

$\mathrm{E}$

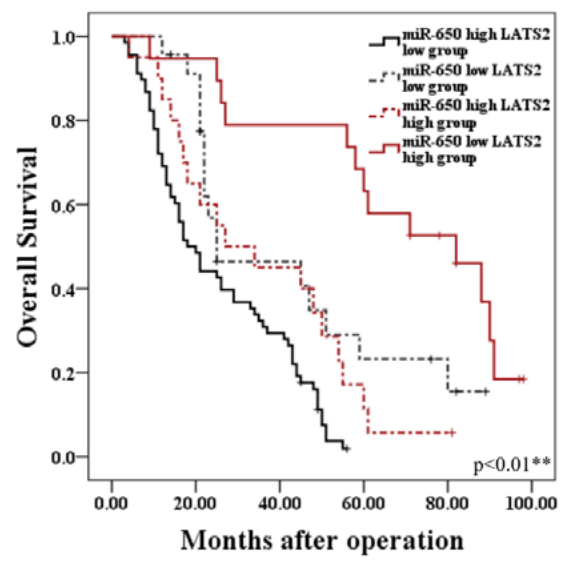

B

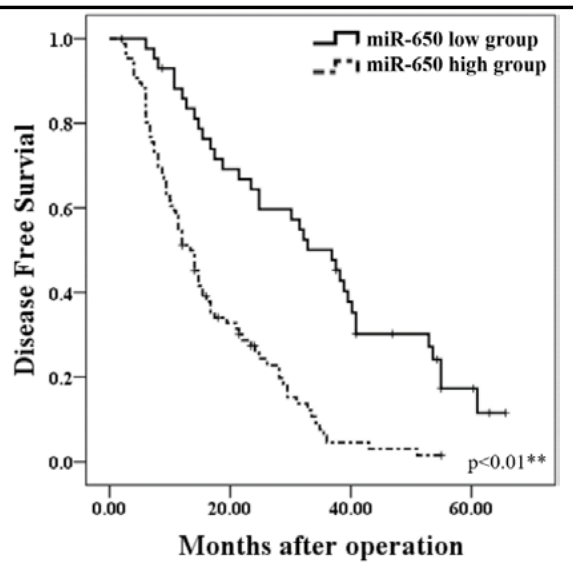

$\mathrm{D}$

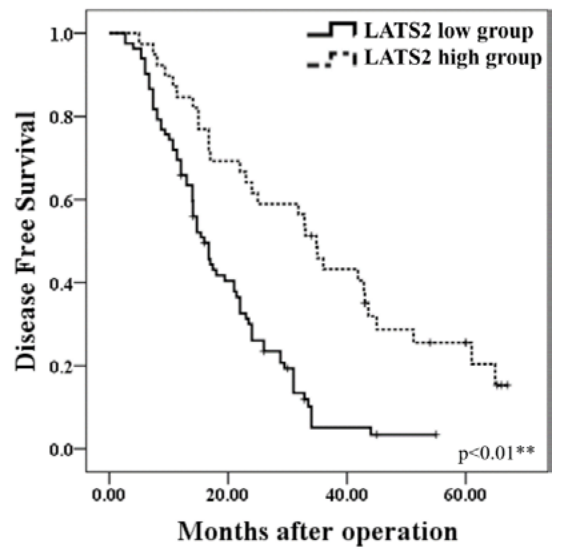

$\mathrm{F}$

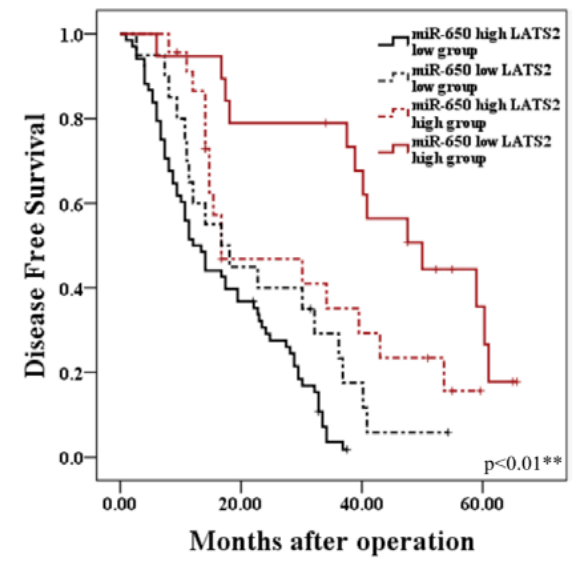

Fig. 9. Prognostic value of miR-650 and LATS2 expression for HCC patients as determined by a KaplanMeier analysis. A. Comparison of OS between HCC patients with high and low miR-650 expression levels. B. Comparison of DFS between HCC patients with high and low miR-650 expression levels. C. Comparison of OS between HCC patients with high and low LATS2 expression levels. D. Comparison of DFS between HCC patients with high and low LATS2 expression levels. E. Comparison of OS among four subgroups of HCC patients (high miR-650/low LATS2 expression; low miR-650/low LATS2 expression; high miR-650/high LATS2 expression; low miR-650/high LATS2 expression). F. Comparison of DFS among four subgroups of HCC patients (high miR-650/low LATS2 expression; low miR-650/low LATS2 expression; high miR-650/ high LATS2 expression; low miR-650/high LATS2 expression) (** $<0.01)$. 


\section{Cellular Physiology Cell Physiol Biochem 2018;51:1179-1192 and Biochemistry \begin{tabular}{l|l} 
DOI: 10.1159/000495495 & $\begin{array}{l}\text { (c) } 2018 \text { The Author(s). Published by S. Karger AG, Basel } \\
\text { www.karger.com/cpb }\end{array}$
\end{tabular} \\ Han et al.: miR-650 Promotes the EMT of HCC by Directly Inhibiting LATS2}

\section{Discussion}

In this study, we demonstrated for the first time that miR-650 promotes HCC metastasis and EMT by directly targeting LATS2. MiR-650 reportedly helps mediate the progression of various cancers [8-10]. However, the role of miR-650 appears to differ depending on the type of cancer. Therefore, the effects and clinical significance of miR-650 in HCC as well as the underlying mechanisms should be further characterized. Our data from in vivo (clinical tissue samples) and in vitro analyses revealed novel information regarding the role of miR650 in HCC progression.

miRNAs are commonly involved in the regulation of invasion and metastasis in various cancers $[17,18]$. Most HCC patient deaths are due to recurrence and metastasis. Thus, we were interested in exploring whether miR-650 influences the progression of metastasis in HCC. Our examination of clinical tissue samples indicated that high miR-650 expression levels are associated with microscopic vascular invasion and the advanced TNM stage of HCC. Additionally, an in vitro assay involving Transwell plates revealed that miR-650 promotes HCC cell migration and invasion. There is accumulating evidence that miRNAs have a crucial function related to the progression of $\operatorname{HCC}$ EMT $[19,20]$. Considering the EMT is a wellknown basal mechanism related to cancer cell migration and invasion [3], we speculated that miR-650 may influence HCC EMT. Our western blot assay results suggested that decreased miR-650 levels increased epithelial marker expression and decreased mesenchymal marker expression, while increased miR-650 levels had the opposite effects. Moreover, an IHC assay involving HCC tissues further demonstrated that the miR-650 expression level is negatively associated with E-cadherin expression but is positively correlated with vimentin expression. Our results confirmed the previously reported aberrant expression of miR-650 in HCC tissues and its association with HCC progression [12]. Moreover, we further established the relationship between miR-650 and HCC EMT.

The Hippo signaling pathway regulates the biological behavior of multiple solid tumor cells [21]. Additionally, the function of the LATS2/YAP signal on the EMT of cancers, including HCC, has been described. In this study, we observed that LATS2 is directly targeted by miR650. A recent study involving NSCLC cells indicated that miR-650 promotes cell migration and invasion by targeting LATS2 [22]. Our in vitro and in vivo analyses support these published findings, while also demonstrating that LATS2 counteracts the effects of miR650 on HCC cell proliferation, migration, and invasion. Moreover, miR-650 inhibits LATS2 expression, leading to the suppression of YAP and its downstream target genes. Therefore, miR-650 may promote HCC EMT by targeting LATS2. However, it is important to note that LATS2 is not the only gene directly targeted by miR-650. A recent investigation concluded that miR-650 inhibits the progression of high-risk, non-metastatic colorectal cancer by suppressing the AKT2/GSK3 $\beta /$ E-cadherin pathway [23]. It was also suggested that miR-650 can decrease the sensitivity of lung adenocarcinoma cells to docetaxel by targeting $B c l-2$ / $B a x$ [24]. It is unknown whether genes such as $A K T 2$ and $B c l-2 / B a x$ are also targeted by miR650 in HCC. Furthermore, among the genes targeted by miR-650, it is unclear whether LATS2 is the most important determinant for the effects of miR-650 on HCC metastasis and EMT. The mechanisms underlying miR-650 functions related to HCC progression will need to be thoroughly investigated.

Many miRNAs may be relevant as diagnostic and prognostic biomarkers for HCC patients $[25,26]$. We herein provide data for clinical samples obtained from 130 HCC patients, which revealed that high miR-650 levels and low LATS2 levels are associated with unfavorable clinical features and poor outcomes. These results may be useful for determining the prognosis for HCC patients. We also observed that miR-650 promotes HCC metastasis and EMT by directly inhibiting LATS2 expression. Therefore, targeting the miR-650/LATS2 pathway may represent a new therapeutic approach for HCC patients. 


\section{Cellular Physiology Cell Physiol Biochem 2018;51:1179-1192 and Biochemistry \begin{tabular}{l|l} 
DOI: 10.1159/000495495 2018 The Author(s). Published by S. Karger AG, Basel \\
Publion
\end{tabular}

\section{Conclusion}

Our findings revealed the novel clinical and biological significance of miR-650 in HCC. Furthermore, the miR-650/LATS2 pathway may be useful as a biomarker and potential therapeutic target for HCC patients.

\section{Abbreviations}

EMT (epithelial-mesenchymal transition); HCC (hepatocellular carcinoma); miRNA (MicroRNA); IHC (immunohistochemistry); GAPDH (glyceraldehyde 3-phosphate dehydrogenase); LATS2 (large tumor suppressor kinase 2); FBS (fetal bovine serum); YAP (yes-associated protein); OS (overall survival); DFS (disease-free survival).

\section{Acknowledgements}

Li li Han collected the clinical samples and performed most of the experiments. Xiao ran Yin performed RT-qPCR assay and the statistical analysis. Shu qun Zhang designed the study and assisted in the drafting of the manuscript. All authors have read and approved the final manuscript. The authors would like to thank Professor Chen Huang of Xi'an Jiaotong University (Xi'an, China) for providing the experimental platform and expert opinions.This study was supported by a grant from the Natural Science Basic Research Plan in Shaanxi Province of China (No. 1191329734) and China Postdoctoral Science General Financial Grant (no. 2017M623193).

\section{Disclosure Statement}

All authors declare they have no competing interests.

\section{References}

1 Finn RS: Current and future treatment strategies for patients with advanced hepatocellular carcinoma: role of mTOR inhibition. Liver Cancer2012;3:247-256.

2 Kew MC: Epidemiology of chronic hepatitis B virus infection, hepatocellular carcinoma, and hepatitis B virus-induced hepatocellular carcinoma. Pathol. Biol 2010;58:273-274.

3 Mathias RA, Gopal SK, Simpson RJ: Contribution of cells undergoing epithelial-mesenchymal transition to the tumour microenvironment. J Proteomics2013;78:545-557.

-4 Zhang LY, Liu M, Li X: miR-490-3p modulates cell growth and epithelial to mesenchymal transition of hepatocellular carcinoma cells by targeting endoplasmic reticulum-golgi intermediate compartment protein 3 (ERGIC3). J Biol Chem2013;288:4035-4047.

-5 Huang XH, Wang Q, Chen JS: Bead-based microarray analysis of microRNA expression in hepatocellular carcinoma: miR-338 is down-regulated. Hepatol Res2009;39:786-794.

6 Zimmerman AL, Wu S: MicroRNAs, cancer and cancer stem cells. Cancer Lett2011;300: 10-19.

7 Feng L, Xie Y, zhang H: Down-regulation of NDRG2 gene expression in human colorectal cancer involves promoter methylation and microRNA-650. Biochem Biophys Res Commun 2011; 406: 534-538.

-8 Zuo H, Yu YP, Ding Y: Oncogenic activity of miR-650 in prostate cancer is mediated by suppression of CSR1 expression. Am J Pathol 2015; 185: 1991-1999.

-9 Das S: Evolutionary origin and genomic organization of micro-RNA genes in immunoglobulin lambda variable region gene family. Mol Biol Evo 2009;126:1179-1189.

10 Mraz M, Dolezalova D, Plevova K: MicroRNA-650 expression is influenced by immunoglobulin gene rearrangement and affects the biology of chronic lymphocytic leukemia. Blood 2012;119: 2110-2113. 


\section{Cellular Physiology Cell Physiol Biochem 2018;51:1179-1192

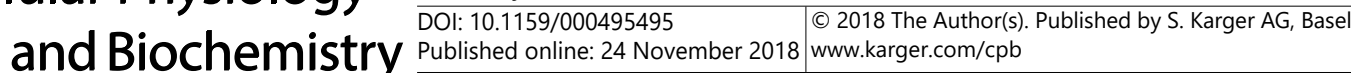

Han et al.: miR-650 Promotes the EMT of HCC by Directly Inhibiting LATS2

11 Zhang X, zhu W, zhang J: MicroRNA-650 targets ING4 to promote gastric cancer tumorigenicity. Biochem Biophys Res Commun 2010;395: 275-280.

12 Zeng ZL, Li FJ, Gao F: Upregulation of miR-650 is correlated with down-regulation of ING4 and progression of hepatocellular carcinoma. J Surg Oncol2013;107:105-111.

13 Han LL, Nan HC, Tian T: Expression and significance of the novel tumor-suppressor gene SMG-1 in hepatocellular carcinoma. Oncol Rep 2014;31:2569-2578.

14 Hao Y, Chun A, Cheung K:Tumor suppressor LATS is a negative regulator of oncogene YAP. The Journal of biological chemistry 2008; 283:5496-5509.

15 Guo C, Wang X, Liang L: LATS2-mediated YAP1 phosphorylation is involved in HCC tumorigenesis. Int J Clin Exp Pathol 2015; 8:1690-1697.

16 Chen L, Loh PG, Song H: Structural and functional insights into the TEAD-YAP complex in the Hippo signaling pathway. Protein Cell 2010;1:1073-1083.

17 Gong F, Ren P, Zhang Y: MicroRNAs-491-5p suppresses cell proliferation and invasion by inhibiting IGF2BP1 in non-small cell lung cancer.Am J Transl Res 2016; 8: 485-495.

18 Fite K, Gomez-Cambronero J: Down-regulation of MicroRNAs (MiRs) 203, 887, 3619 and 182 Prevents Vimentin-triggered, Phospholipase D (PLD)-mediated Cancer Cell Invasion. J Biol Chem 2016;291:719-730.

19 Jaca A, Govender P, Locketz M: The role of miRNA-21 and epithelial mesenchymal transition process in colorectal cancer. J Clin Pathol 2017;70: 331-356.

20 Tang 0, Chen XM, Shen S: MiRNA-200b represses transforming growth factor- $\beta 1$-induced EMT and fibronectin expression in kidney proximal tubular cells. Am J Physiol Renal Physiol 2013; 304: 1266-1273.

21 Murakami H, Mizuno T, Taniguchi T: LATS2 is a tumor suppressor gene of malignant mesothelioma. Cancer Res 2011;71:873-883.

-22 Ye Y, Zhuang J, Wang G: MicroRNA-650 promotes cell proliferation, migration and invasion in non-small cell lung cancer by directly targeting LATS2. Exp Ther Med 2017;14:867-873.

-23 Zhou C, Cui F, Li J: MiR-650 represses high-risk non-metastatic colorectal cancer progression via inhibition of AKT2/GSK3 $\beta /$ E-cadherin pathway. Oncotarget 2017; 25:49534-49547.

24 Huang JY, Cui SY, Chen YT: MicroRNA-650 was a prognostic factor in human lung adenocarcinoma and confers the docetaxel chemoresistance of lung adenocarcinoma cells via regulating Bcl-2/Bax expression. PLoS One 2013;8:72615.

25 Mirzaei HR, Sahebkar A, Mohammadi M: Circulating microRNAs in Hepatocellular Carcinoma: Potential Diagnostic and PrognosticBiomarkers. Curr Pharm Des 2016;22:5257-5269.

26 Gougelet A, Colnot S: Hepatocellular carcinoma diagnosis: Circulating microRNAs emerge as robust biomarkers. Clin Res Hepatol Gastroenterol 2016;40: 367-369. 